\title{
Small-scale dipolarization fronts in the Earth's magnetotail
}

\author{
Jing Huang', Meng Zhou ${ }^{1}$, HuiMin Li ${ }^{2}$, XiaoHua Deng ${ }^{1}$, Jiang Liư ${ }^{3}$, and ShiYong Huang4 \\ ${ }^{1}$ Institute of Space Science and Technology, Nanchang University, Nanchang 330031, China; \\ 2School of Physics and Optoelectronic Engineering, Xidian University, Xi'an 710071, China; \\ ${ }^{3}$ Department of Earth, Planetary, and Space Sciences and Institute of Geophysics and Planetary Physics, University of California, Los Angeles, California, USA; \\ ${ }^{4}$ School of Electronic and Information, Wuhan University, Wuhan 430072, China
}

\begin{abstract}
Previous studies suggest that dipolarization fronts (DFs) are 1 to $3 R_{\mathrm{E}}$ ( $R_{\mathrm{E}}$ is the earth radius) wide in the dawn-dusk direction. Recent kinetic simulations have found that DFs may break up into small-scale structures after they are produced by reconnection. Motivated by this simulation, we revisited the scale size of DFs in the dawn-dusk direction by using Cluster observations during the years when the inter-distance among Cluster spacecraft was between 1000 and $2000 \mathrm{~km}$. We selected the DFs that were detected by more than one spacecraft and estimated the radii of these DFs by a simple geometrical analysis, which is based on comparison of DF normals observed by different spacecraft. We found a few DFs that were only a few ion inertial lengths in the dawn-dusk direction. These results point out the importance of multi-scale coupling during the evolution of DFs.
\end{abstract}

Keywords: dipolarization front; small scale; Earth's magnetotail

Citation: Huang, J., Zhou, M., Li, H. M., Deng, X. H., Liu, J., and Huang, S. Y. (2019). Small-scale dipolarization fronts in the Earth's magnetotail. Earth Planet. Phys., 3(4), 358-364. http://doi.org/10.26464/epp2019036

\section{Introduction}

Magnetospheric substorms are one of the most important explosive phenomena in space weather. One crucial substorm ingredient is the dipolarization front (DF), which refers to the transient increase of magnetic field $B_{z}$ embedded within earthward fast flows in the magnetotail (e.g., Nakamura et al., 2002; Ohtani et al., 2004; Runov et al., 2009). A DF is a boundary layer separating an earthward moving depleted flux tube that contains hot tenuous plasmas and an ambient flux tube that contains cold dense plasmas (Runov et al., 2011). It is also a thin current layer where the Hall electric field normal to the front has been observed (Zhou $M$ et al., 2009; Fu HS et al., 2012a). DFs are pivotal in the dynamics of Earth' s magnetotail. Liu J et al. (2014) found that DFs carry significant magnetic flux in the magnetotail. It is suggested that DFs are major sites for energy conversion and particle acceleration in the near-Earth magnetotail (Huang SY et al., 2012, 2015a; Angelopoulos et al., 2013; Deng XH et al., 2010; Ashour-Abdalla et al., 2011; Fu HS et al., 2011; Wang $Y$ et al., 2015). Moreover, DFs are usually accompanied by different plasma waves, from the ion cyclotron frequency to above the electron cyclotron frequency, that substantially affect the dynamics of particles near the DFs (Deng XH et al., 2010; Zhou M et al., 2009, 2013, 2014; Li HM et al., 2015).

The normal-direction thickness of most DFs is on the order of the ion inertial length or ion Larmor radius (Runov et al., 2009; Zhou M

Correspondence to: M. Zhou, mengzhou@ncu.edu.cn

Received 02 JAN 2019; Accepted 09 MAY 2019.

Accepted article online 22 MAY 2019.

(C) 2019 by Earth and Planetary Physics. et al., 2009); the widths of DFs along their dawn-dusk (cross-tail) direction is generally believed to be of the fluid scale, which is much larger than the ion characteristic scales. Sergeev et al. (1996) found that depleted flux tubes, the leading edges of which resemble DFs, are $1-2 R_{\mathrm{E}}$ wide in the dawn-dusk direction in the near-Earth tail. This is similar to the inferred width of fast earthward flows in the magnetotail (Nakamura et al., 2004). Recent multi-spacecraft analysis using THEMIS and Cluster observations have confirmed the previous results that DF width is a few (1-3) $R_{\mathrm{E}}$ in the dawn-dusk direction (Liu J et al., 2013; Huang SY et al., 2015b).

Recent 3-D kinetic simulation has suggested that DFs may break up to form multiple small-scale (a few ion inertial lengths) DFs along the tangential surface of the front (Pritchett et al., 2014, 2016). Energy conversion and dissipation at a DF mainly occurs in these ion-scale small structures. Moreover, Zhou M et al. (2017) studied a three-dimensional X-line in the near-Earth magnetotail. They found that two spacecraft that were separated by $1 R_{\mathrm{E}}$ along the dawn-dusk direction observed different magnetic structures (one observed flux ropes and the other one observed DFs) near the diffusion region. This implies that the DFs had dawn-dusk sizes much smaller than $1 R_{\mathrm{E}}$ close to the $\mathrm{X}$-line.

Motivated by the above-mentioned simulations and observations, we revisited the dawn-dusk sizes of the DFs in the near-Earth tail by using data from the Cluster mission in the years 2001, 2002, and 2004 (Escoubet et al., 1997), years during which the four Cluster spacecraft kept a tetrahedron shape in space with spacing that ranged from 1000 to $2000 \mathrm{~km}$. This spacing enabled Cluster to uncover the ion-scale DFs or the ion-scale structures developed on DFs that cannot be resolved by THEMIS, or by Cluster 
after the year 2005, when the spacing among these spacecrafts was generally larger than 5000 km (e.g., Liu J et al., 2013; Huang SY et al., 2015b). Data from the Fluxgate magnetometer (FGM) (Balogh et al., 2001), Electric Field and Waves (EFW) (Gustafsson et al., 2001) and Cluster lon Spectrometry (CIS) (Rème et al., 2001) instruments on board Cluster were used in this study. We should note that the CIS instrument on C2 has been out of order since 2001, hence ion plasma data were available only from the other three spacecraft. We used the spacecraft potential provided by the EFW instrument to monitor rapid variations of plasma density (Pedersen et al., 2008). Although the spacecraft potential cannot always provide an accurate value for the plasma density, its variations are a good indicator of density change.

\section{Database and Methodology}

In total, we selected 96 DFs observed by Cluster in the near-Earth magnetotail during the years of 2001, 2002, and 2004. These DFs are from the database in Li HM et al. (2015), which includes a total of 282 DFs. The 96 chosen for this study were identified by using the following criteria:

(1) The spacecraft was in the plasma sheet, i.e., plasma $\beta \geq 0.5$ and $\left|B_{x}\right|<20 \mathrm{nT}$.

(2) The observed increase of $B_{z}$ was rapid, i.e., $\Delta B_{z}>10 \mathrm{nT}$ in $16 \mathrm{~s}$.

(3) The magnetic field disturbance before the DF was small, i.e., variance of the fluctuations was less than $3 \mathrm{nT}$ in 3 mins before the arrival of the DF.

These criteria are similar to those used in previous statistical studies of DFs (Schmid et al., 2011; Fu HS et al., 2012b; Liu J et al., 2013).

To calculate the radii of DFs using our multi-spacecraft method, we further require that the DF must be observed by more than one spacecraft - that is, the time delays between DF data collected by different spacecraft should be less than $20 \mathrm{~s}$.

We employed the same geometry analysis as Liu J et al. (2013) to estimate the dawn-dusk sizes of the DFs. We assumed that a DF is a semicircular structure in the $X-Y$ plane, in geocentric solar magnetosphere (GSM) coordinates, and that it is symmetric with respect to the propagation direction of the DF (See Figure 1). We repositioned each DF into a local orthogonal coordinate system $X^{*} Y^{*} Z^{*}$ by rotating the GSM coordinate system about the $Z_{\text {gsm }}$ axis by a certain angle $\varphi=\tan ^{-1}\left(\frac{V_{B, y}}{V_{B, x}}\right)$, where $V_{B}$ is the field line velocity $V_{B}=E \times B / B^{2}$ (Liu J et al., 2013), which has been averaged over all the spacecraft that encountered the DF. $X^{*}$ is along the projection of $V_{B}$ in the $X_{\mathrm{gsm}}-Y_{\mathrm{gsm}}$ plane, $Z^{*}=Z_{\mathrm{gsm}}$ and $Y^{*}=Z^{*} \times X^{*}$. Minimum variance analysis (MVA) of the magnetic fields (Sonnerup and Scheible, 1998) is used to determine the normal orientation $N$ of the DFs at the contact point of the spacecraft trajectory and the DF. The ratio between the intermediate eigenvalue $\lambda_{2}$ and the minimum eigenvalue $\lambda_{3}$ is used to evaluate the accuracy of the MVA. Here we require that $\lambda_{2} / \lambda_{3}$ must be larger than 10 to provide a reliable normal direction. If $\lambda_{2} / \lambda_{3}$ is less than 10 , then the observation from this spacecraft will be discarded. Moreover, $N_{x}^{*}$ is always set as positive.

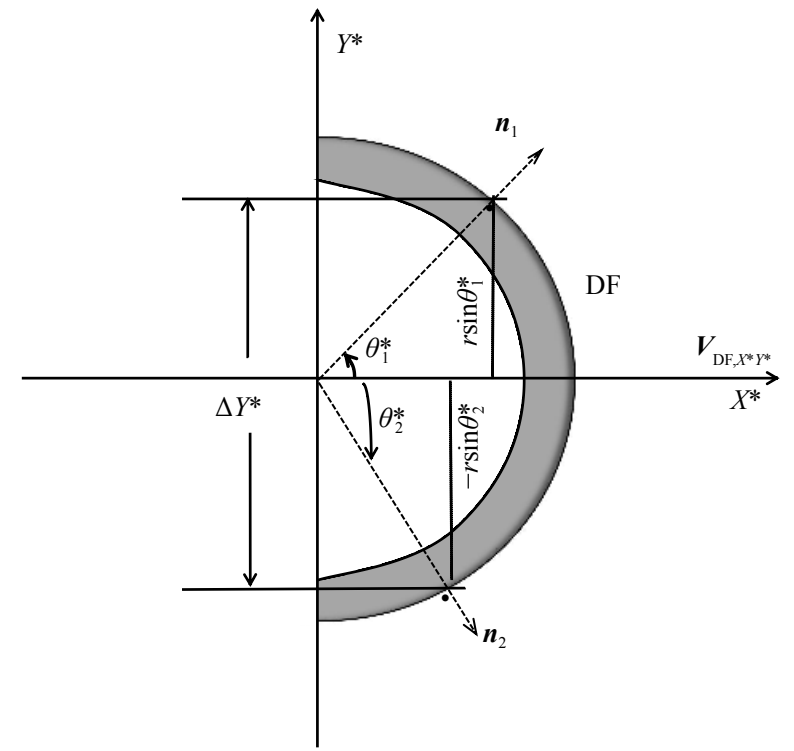

Figure 1. Demonstration of how to estimate the radius of the DF (assuming it is a semi-circle in the $X^{*} Y^{*}$ plane) by two spacecraft observations. $\boldsymbol{n}_{1}$ and $\boldsymbol{n}_{2}$ are the normals of DF at the two contact points between the two spacecraft and the DF layer. $X^{*}$ is in the direction of the DF's convective velocity in the $X_{\mathrm{gsm}}-Y_{\mathrm{gsm}}$ plane, while $Y^{*}$ is orthogonal to $X^{*}$ and lies in the $X_{\mathrm{gsm}}-Y_{\mathrm{gsm}}$ plane.

The DF radius, $r$, can be estimated from a dual observation by the following equation. Here $\Delta Y^{*}$ is the separation of the two spacecraft in the $Y^{*}$ direction, and the azimuthal angle, $\theta^{*}$, between the $\mathrm{DF}$ normal and the $X^{*}$ axis is given by $\theta^{*}=\tan ^{-1}\left(N_{\mathrm{DF}, y^{*}} / N_{\mathrm{DF}, x^{*}}\right)$, where $N_{D F}$ is the normal of the DF estimated by each spacecraft.

$$
r=\left|\frac{\Delta Y^{*}}{\sin \theta_{1}^{*}-\sin \theta_{2}^{*}}\right| \text {. }
$$

If more than two spacecraft detected the DF and provided reliable normal direction measurements, for the calculation we chose data from the two spacecraft that were farthest from each other in the $Y^{*}$ direction. We further limited the analysis to data from spacecraft pairs for which $\Delta \theta^{*}>40^{\circ}$, in order to avoid the possibility of large errors caused by observations from an insufficient angular difference. If data from the two chosen spacecraft inferred a concave geometry, we made a second calculation of $r$, based on data from a second pair of spacecraft, the pair that were the second farthest from each other in the $Y^{*}$ direction and that also satisfied $\Delta \theta^{*}>40^{\circ}$. We repeated this procedure until we found a convex shape. If we could not find a convex shape for a particular event, we discarded the event.

The errors associated with eigenvectors provided by the MVA can be estimated as (Sonnerup and Scheible, 1998):

$$
\left|\Delta \alpha_{i j}\right|=\sqrt{\frac{\lambda_{3}}{M-1} \cdot \frac{\lambda_{i}+\lambda_{j}-\lambda_{3}}{\left(\lambda_{i}-\lambda_{j}\right)^{2}}}, i \neq j=1,2,3
$$

where $\Delta a_{i j}$ is the angular deviation of vector $n_{i}$ toward vector $n_{j}, M$ is the number of data points used for MVA, and $\lambda_{1}, \lambda_{2}$ and $\lambda_{3}$ are the maximum, intermediate, and minimum eigenvalues, respectively. $\Delta a_{31}$ and $\Delta a_{32}$ denote the angular errors of the DF's normal, 
estimated by MVA. For each spacecraft, we obtain four vectors that corresponding to $\Delta a_{31}$ and $\Delta a_{32}$, and four corresponding $\theta^{*}$ in equation (1). Two spacecraft will result in 16 different values of $r$, based on which we can obtain the diameter of the DF and the error associated with the calculated diameter.

\section{Multiple Cases Study}

Below we show two examples of small-scale DFs detected by Cluster. Figure 2 shows the four spacecraft observations of a DF between 23:11:00 and 23:14:00 UT on August 07, 2004, when Cluster was near $[-16.2,-8.9,0.3] R_{\mathrm{E}}$ in the GSM coordinates. The four Cluster spacecraft formed a regular tetrahedron in space with a maximum inter-distance about $1200 \mathrm{~km}$. A transient increase of $B_{z}$ from 0 to $14 \mathrm{nT}$ was recorded by Cluster at around 23:12:20 UT. A small $B_{z}$ dip from 2 to $-1 \mathrm{nT}$ ahead of the DF was also observed (Figure $2 \mathrm{c}$ ). The duration of this DF (from the negative $B_{z}$ peak to the first positive peak) was about $8 \mathrm{~s}$. This DF was observed away from the neutral sheet as $B_{x}$ changed from 15 to $5 \mathrm{nT}$ associated with the DF. This DF was embedded within an earthward flow with a peak speed near $400 \mathrm{~km} / \mathrm{s}$ (Figure 2e), and was accompanied by a drop in the plasma density observed by $\mathrm{C} 1$ and $\mathrm{C} 4$ and an enhancement in the plasma density observed by $\mathrm{C} 2$ and $\mathrm{C} 3$, probably because C2 and C3 approached the neutral sheet more deeply than the other two spacecraft. These features are consistent with previous observations of DFs in the near-Earth plasma sheet (e.g., Zhou M et al., 2009).

We used MVA to determine the normal directions of the DF at the contact point of the DF and the spacecraft trajectory. The MVA results, spacecraft relative positions, and normal angles with respect to the $X^{*}$ axis, are summarized in Table 1 . Since $\lambda_{2} / \lambda_{3}$ is less than 10 for $\mathrm{C} 3$, we do not use the measurement from $\mathrm{C} 3$ to calculate the radius of the DF. Following the procedure depicted in the above section, we evaluated the radius of this DF. The moving velocity of the DF is given by $V_{B}=E \times B / B^{2} \approx(210,-148,31) \mathrm{km} / \mathrm{s}$. It means that the DF moved primarily in the $X-Y$ plane, its velocity primarily earthward and dawnward. This DF's moving velocity is quite different from the ion bulk velocity, suggesting that the DF was probably not frozen-in to the ions. We estimated the DF thickness by $V_{B} \cdot N_{D F} d t$, where $N_{D F}$ is the normal of the DF and $d t$ is the duration of the DF. Since the normals measured by different spacecraft were different, the corresponding thicknesses of the DF

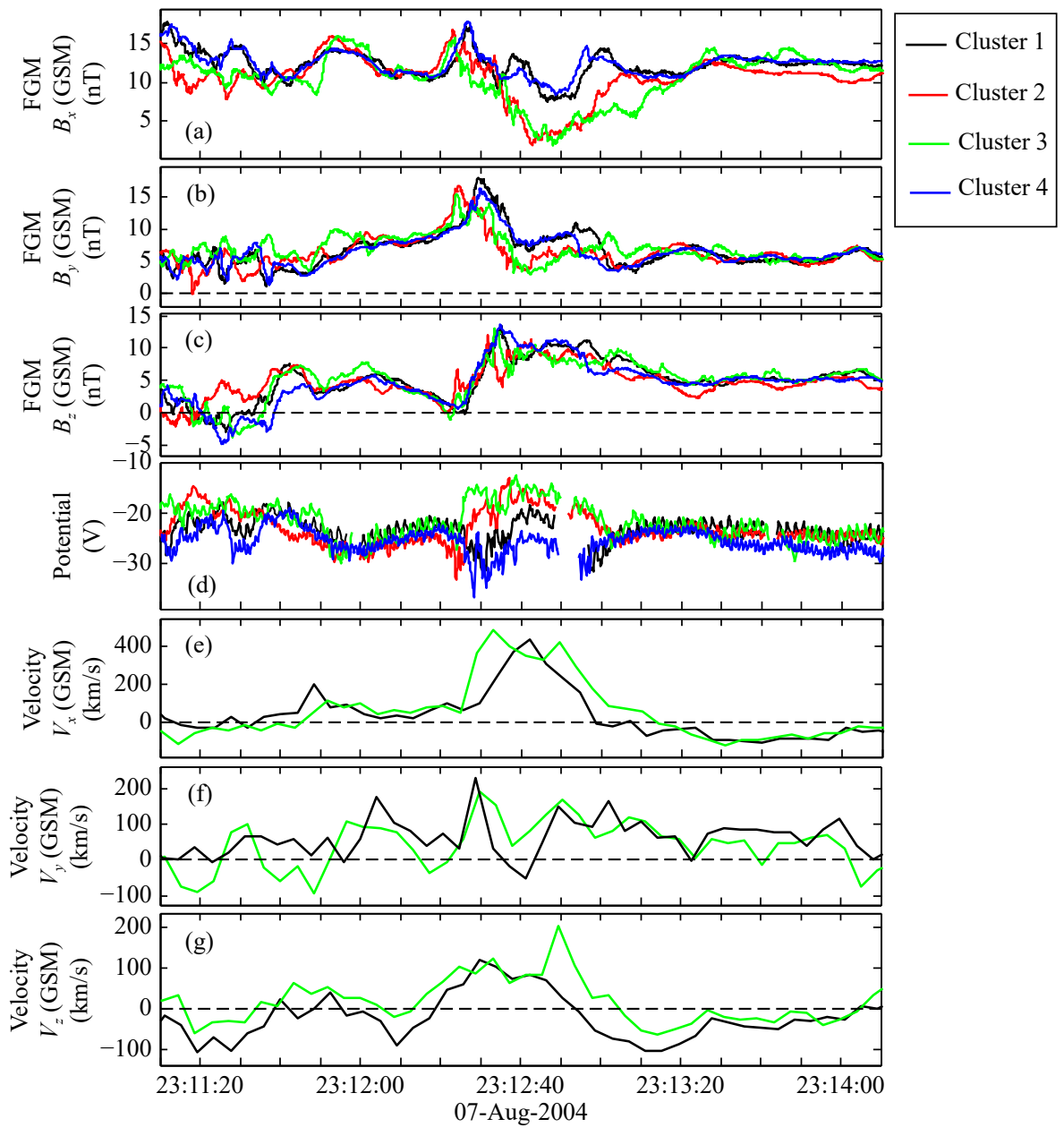

Figure 2. Overview of a DF observed by the four Cluster spacecraft between 23:11:00 and 23:14:00 UT on August 07, 2004. From the top to the bottom: (a, b, and c) $X, Y$, and $Z$ components of the magnetic field; (d) spacecraft potential; (e, $\mathrm{f}$, and g) $X, Y$, and $Z$ components of the ion bulk velocity from $C 1$ and C3. The CIS instrument on board C2 has been out of order since 2001, hence no ion measurement data were available from C2. Similarly, no C4 ion moment data were included because the CIS-HIA instrument on board C4 is out of order. 
Table 1. Normals estimated by MVA, ratio between the intermediate and minimum eigenvalues $\lambda_{2} / \lambda_{3}$, probe relative position, and the angle between the DF normal and the $X^{*}$ axis. $\mathrm{C} 1$ is set as the origin in calculating the relative spacecraft positions. The vectors are displayed in the $X^{*} Y^{*} Z^{*}$ coordinates. The results are for the August 07, 2004 event

\begin{tabular}{ccccc}
\hline SC & $N^{*}$ & $\lambda_{2} / \lambda_{3}$ & Relative position in the $X^{*} Y^{*} Z^{*}$ coordinates $(\mathrm{km})$ & $\theta^{*}\left({ }^{\circ}\right)$ \\
\hline 1 & $0.89,-0.11,0.45$ & 15.3 & $0,0,0$ & 28 \\
2 & $0.79,-0.23,0.57$ & 13 & $-1050,-60,-320$ & $-70,-310,-1080$ \\
3 & $0.89,0.09,0.49$ & 4.3 & $-20,-790,-240$ & -17 \\
4 & $0.52,-0.66,0.54$ & 10.5 & - \\
\hline
\end{tabular}

were also different. The thicknesses of this DF, as measured by data from $\mathrm{C} 1, \mathrm{C} 2$, and $\mathrm{C} 4$, respectively, are approximately $1720 \mathrm{~km}-2.4 d_{\mathrm{i}}, 2170 \mathrm{~km}-3 d_{\mathrm{i}}$ and $2230 \mathrm{~km}-3.1 d_{\mathrm{i}}$, where $d_{\mathrm{i}}(\sim 720 \mathrm{~km})$ is the ion inertial length in the ambient plasma sheet before the arrival of the DF. This implies that the thickness of the DF was not uniform along its tangential surface. Knowing the convection speed of this DF's $V_{B}$, we then transform the vectors into the DF local coordinates $X^{*} Y^{*} Z^{*}$. Applying equation (1) to the observations from $\mathrm{C} 1$ and $\mathrm{C} 4$, we find that the DF was a convex shape in the $X^{*} Y^{*}$ plane, i.e., $\Delta N_{y, \mathrm{Cl}-\mathrm{C} 4}^{*} / \Delta Y_{\mathrm{Cl}-\mathrm{C} 4}^{*}>0$. The angular errors of the DF's normal observed by $C 1$ are: $\Delta a_{31}= \pm 0.70^{\circ}, \Delta a_{32}= \pm 1.96^{\circ}$, and by $C 4$ are: $\Delta a_{31}= \pm 0.69^{\circ}, \Delta a_{32}= \pm 4.48^{\circ}$. The computed diameter of this DF is, therefore, $2130 \pm 338 \mathrm{~km}-2.8 \pm 0.4 d_{\mathrm{i}}$. This diameter is much smaller than that of the DFs evaluated by THEMIS (e.g., Liu J et al., 2013). It is also much smaller than those of the DFs observed by Cluster in the year 2005 when the inter-distance among Cluster spacecraft was more than 5000 km (Huang SY et al., $2015 b)$. The relative positions of the four spacecraft with respect to the DF is shown in Figure 3 a.

The other event was observed by Cluster on October 13, 2004, when Cluster was located at approximately $[-14.2,8.6,-2.4] R_{\mathrm{E}}$ in the GSM coordinates. The four spacecraft were in a tetrahedronal formation with maximum spacing of approximately $1300 \mathrm{~km}$. This second example DF event differs from the first, above, in that it was observed only by three Cluster spacecrafts (C1, C3, and C4); C2 missed this DF. Figure 4 presents the Cluster spacecraft observations from 07:43:50 to 07:45:50 UT. C3 and C4 detected the DF nearly simultaneously at around 07:44:45 UT, while C1 observed the DF about $7 \mathrm{~s}$ later. The DF was characterized by an abrupt increase of $B_{z}$ of approximately 20 to $30 \mathrm{nT}$. This enhancement of $B_{z}$ was preceded by a $B_{z}$ decrease of $6 \mathrm{nT}$, observed by C3; of $2 \mathrm{nT}$, observed by $C 1$ (the $B_{z}$ decrease observed by $C 4$ was minor, only 1 $\mathrm{nT})$. This DF was observed in the southern hemisphere away from the neutral sheet where the average $B_{x}$ was nearly $-15 \mathrm{nT}$. The DF was embedded within an earthward flow with a peak speed around $200 \mathrm{~km} / \mathrm{s}$ (Figure 4e). Similar to the DFs reported in previously published studies, this DF was associated with a sharp plasma density drop, indicated by decreases in spacecraft potential. Table 2 shows the multiple spacecraft analysis results for this DF. The format is the same as in Table 1. $\lambda_{2} / \lambda_{3}$ from C4 was less than 10 , indicating that the DF's normal estimated from $C 4$ data may be unreliable. The propagation speed of this DF's $\mathbf{V}_{B}$ was about $(136,-108,45) \mathrm{km} / \mathrm{s}$. This speed is similar to the ion bulk speed associated with this DF. Based on the propagating speed of the DF, we infer that the thicknesses of the DF were about $580 \mathrm{~km}-1.6 d_{\mathrm{i}}$ and $810 \mathrm{~km}-2.2 d_{\mathrm{i}}$ as measured by $\mathrm{C} 1$ and $\mathrm{C} 3$, respectively, where $d_{\mathrm{i}}(\sim 360 \mathrm{~km})$ is the ion inertial length in the ambient plasma sheet. We used C1 and C3 observations to evaluate the radius of this DF. The angular errors of the DF normal observed by C1 are: $\Delta a_{31}= \pm 0.38^{\circ}, \Delta a_{32}= \pm 0.58^{\circ}$, and by C3 are: $\Delta a_{31}= \pm 0.24^{\circ}$, $\Delta a_{32}= \pm 1.46^{\circ}$. The diameter of this DF is thus estimated to be $1474 \pm 14 \mathrm{~km}=4.2 \pm 0.04 d_{\mathrm{i}}$. In addition, we find that this DF was convex in the $X^{*} Y^{*}$ plane, i.e., $\Delta N_{y, \mathrm{Cl}-\mathrm{C} 3}^{*} / \Delta Y_{\mathrm{Cl} 1 \mathrm{C} 3}^{*}>0$. Figure $3 \mathrm{~b}$ displays the relative positions of the four spacecraft with respect to the DF. It is clear that C2 was far duskward from the other space-
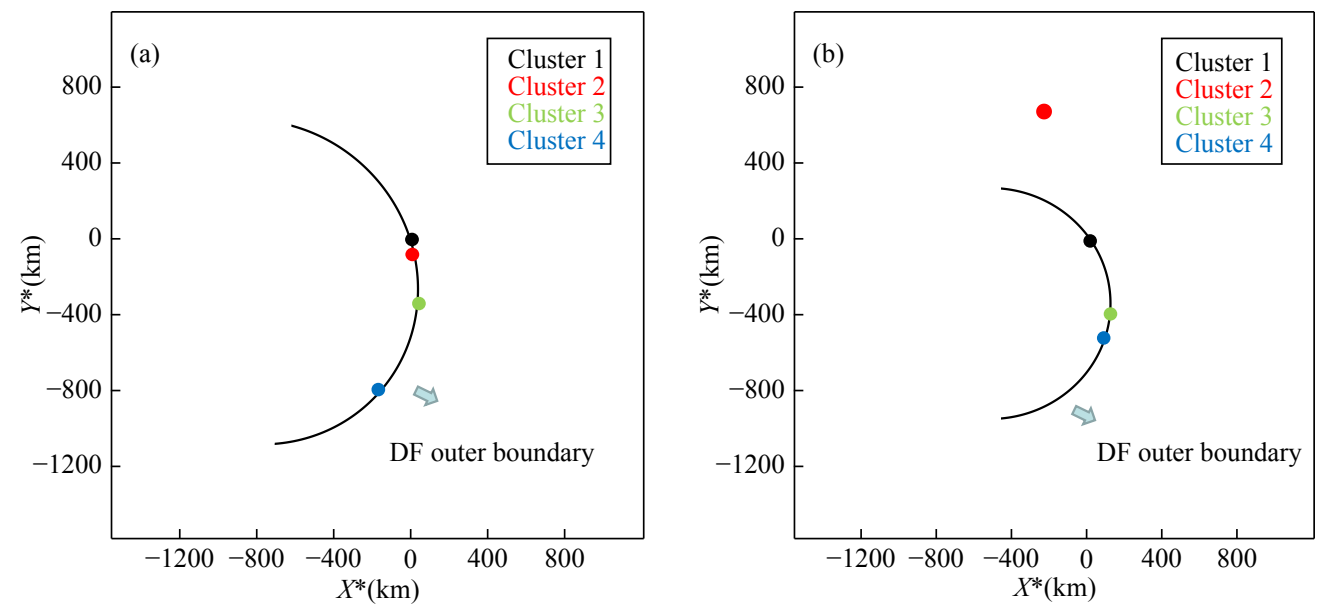

Figure 3. Four Cluster spacecraft positions and DF in the $X^{*} Y^{*}$ plane: (a) at around 23:12 UT on 07 August 2004; (b) at around 07:44 UT on 13 October 2004. The indicated positions of the spacecraft are at the time when they encountered the DF. The position of C2 in panel (b) is obtained at 07:44:50 UT. 


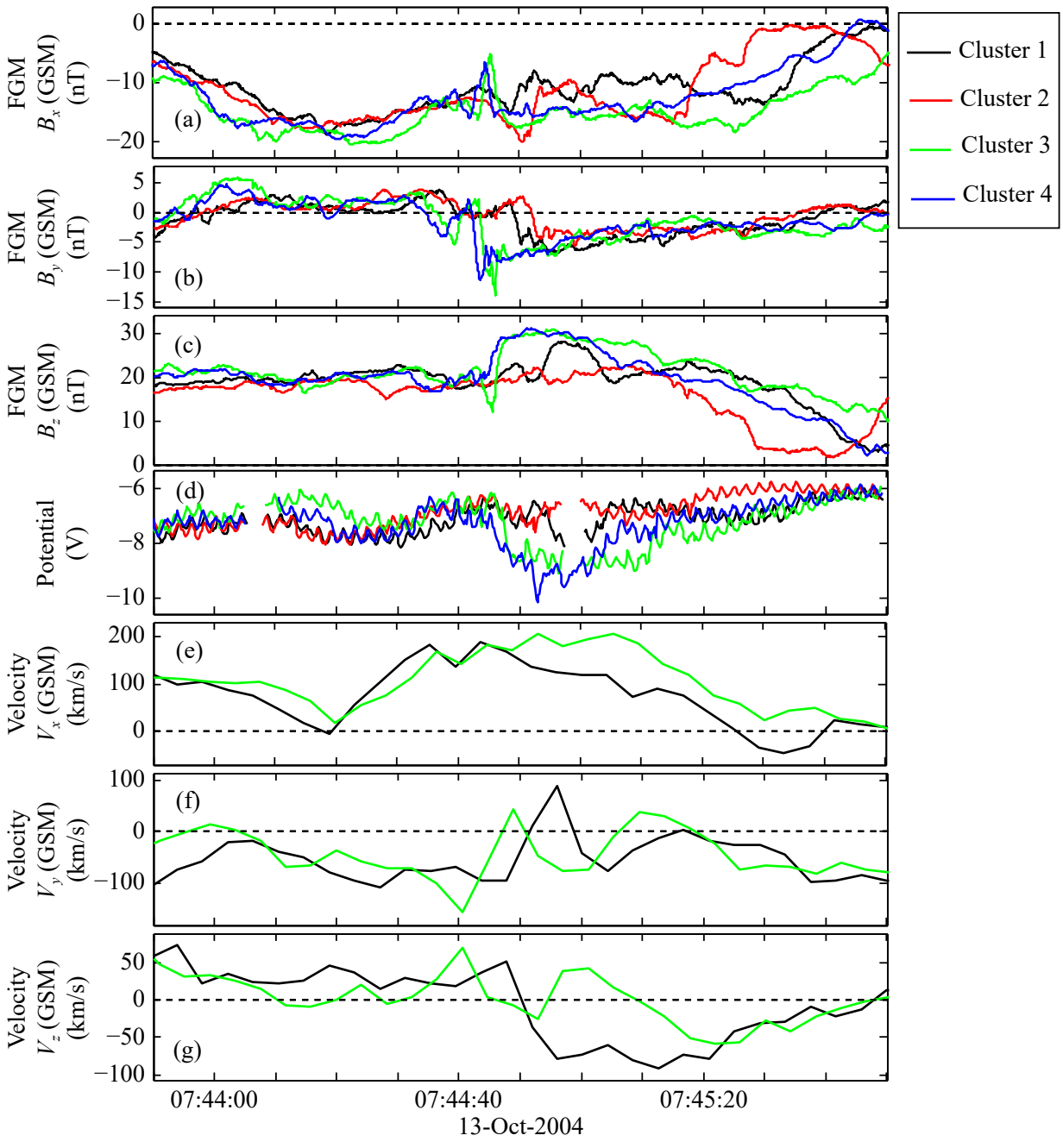

Figure 4. Overview of a DF observed by Cluster between 07:43:50 and 07:45:50 UT on October 13, 2004. The format is same as Figure 2 .

Table 2. The same format as Table 1. This table is for the event of October 13, 2004

\begin{tabular}{ccccc}
\hline SC & $N^{*}$ & $\lambda_{2} / \lambda_{3}$ & $\begin{array}{c}\text { Relative Positions in the } \\
X^{*} Y^{*} Z^{*} \text { coordinates }(\mathrm{km})\end{array}$ & $\theta^{*}\left({ }^{\circ}\right)$ \\
\hline 1 & $0.75,0.55,0.35$ & 35 & $0,0,0$ & 75 \\
2 & - & - & $-230,750,-960$ & - \\
3 & $0.75,-0.31,0.58$ & 43 & $340,-430,-1170$ & 17 \\
4 & $0.82,0.40,0.41$ & 8 & $-510,-500,-680$ & - \\
\hline
\end{tabular}

craft. It missed the DF because the DF was oriented in the dawndusk direction.

We analyzed all of the 96 DFs following the same procedure described above, and found 6 DFs with diameters less than $10 d_{i}$, which is substantially smaller than the dawn-dusk sizes of DFs reported in previous studies (Sergeev et al., 1996; Liu J et al., 2013; Huang SY et al., 2015b). The observation times and the sizes of these DFs are listed in Table 3.

\section{Discussion and Summary}

We have revisited the dawn-dusk scale of earthward propagating
Table 3. List of the small-scale DFs observed by Cluster and their diameters $d$ in the unit of $\mathrm{km}$ and the ion inertial length

\begin{tabular}{cccc}
\hline No & Observation time of the DF $(\mathrm{UT})$ & $d(\mathrm{~km})$ & $d\left(d_{\mathrm{i}}\right)$ \\
\hline 1 & $2001-08-2414: 51: 50$ & $2406 \pm 272$ & $6.60 \pm 0.80$ \\
2 & $2002-09-0205: 27: 55$ & $2340 \pm 12$ & $4.60 \pm 0.04$ \\
3 & $2002-09-3007: 47: 26$ & $2910 \pm 128$ & $9.00 \pm 0.40$ \\
4 & $2004-08-0723: 12: 10$ & $2130 \pm 340$ & $2.80 \pm 0.40$ \\
5 & $2004-08-2619: 11: 28$ & $2886 \pm 142$ & $6.90 \pm 0.34$ \\
6 & $2004-10-1307: 44: 37$ & $1471 \pm 14$ & $4.16 \pm 0.02$ \\
\hline
\end{tabular}

DFs observed by the four Cluster spacecraft during the years 2001, 2002, and 2004, when the inter-distance among Cluster spacecraft was approximately 1000 to $2000 \mathrm{~km}$. By assuming that the DFs were semicircular with symmetry axes along their directions of motion, we estimated the diameters of the DFs and found a few small-scale DFs with diameters on the order of the ion inertial length, which is much smaller than found in previously studies; this result suggests that the dawn-dusk size of the DFs is a few earth radii (e.g., Sergeev et al., 1996; Liu J et al., 2013).

Our result is consistent with those of Pritchett (2016), who found 
that reconnection exhausts break up into small-scale fronts of width $1-2 d_{\mathrm{i}}$ due to the ballooning /interchange-type of instability. On the other hand, Zhou M et al. (2017) found that some DFs originating from reconnection were quite narrow in the out-of-plane direction (in the tail, the out-of-plane direction is close to the dawn-dusk direction). These small-scale DFs may either expand or merge to form larger DFs during their propagation towards Earth (Liu J et al., 2015). We should note that these small-scale structures may be the ripples on DFs, as reported recently in 3-D PIC simulation and magnetospheric multiscale (MMS) observations (Vapirev et al., 2013; Pan DX et al., 2018). These ripples exhibited either ion- or electron-scale wavelengths, and were related to instabilities developed on the front.

Although this study does not reveal the formation mechanism of these ion-scale structures on DFs, it implies that kinetic physics plays an important role in the generation and evolution of DFs. The size in the dawn-dusk direction is important in magnetospheric physics since it determines the magnitude of magnetic flux carried by a DF (Liu J et al., 2014), and potentially affects the energy gain obtained by particles from a DF (Birn et al., 2013). These results are also important for understanding cross-scale coupling in the magnetotail.

\section{Acknowledgments}

This work is supported by the National Natural Science Foundation of China (NSFC) under grant 41774154 and 41504123 , the Science Foundation of Jiangxi Province under grant 20122BAB21 2002, the Nanchang University graduate innovation special fund project under grant CX2017106 and the Key Industry Innovation Chain of Shaanxi under grant 2018JQ4032. Data used in this study were obtained from the Cluster Science Archive (https://csa.esac. esa.int/).

\section{References}

Angelopoulos, V., Runov, A., Zhou, X. Z., Turner, D. L., Kiehas, S. A., Li, S. S., and Shinohara, I. (2013). Electromagnetic energy conversion at reconnection fronts. Science, 341(6153), 1478-1482.

https://doi.org/10.1126/science.1236992

Ashour-Abdalla, M., El-Alaoui, M., Goldstein, M. L., Zhou, M., Schriver, D., Richard, R., Walker, R., Kivelson, M. G., and Hwang, K. J. (2011). Observations and simulations of non-local acceleration of electrons in magnetotail magnetic reconnection events. Nat. Phys., 7(4), 360-365. https://doi.org/10.1038/nphys1903

Balogh, A., Carr, C. M., Acuña, M. H., Dunlop, M. W., Beek, T. J., Brown, P., Fornacon, K. H., Georgescu, E., Glassmeier, K. H., ... Schwingenschuh, K. (2001). The cluster magnetic field investigation: overview of in-flight performance and initial results. Ann. Geophys., 19(10-12), 1207-1217. https://doi.org/10.5194/angeo-19-1207-2001

Birn, J., Hesse, M., Nakamura, R., and Zaharia, S. (2013). Particle acceleration in dipolarization events. J. Geophys. Res.: Space Phys., 118(5), 1960-1971. https://doi.org/10.1002/jgra.50132

Deng, X. H., Ashour-Abdalla, M., Zhou, M., Walker, R., El-Alaoui, M., Angelopoulos, V., Ergun, R. E., and Schriver, D. (2010). Wave and particle characteristics of earthward electron injections associated with dipolarization fronts. J. Geophys. Res.: Space Phys., 115(A9), A09225. https://doi.org/10.1029/2009JA015107

Escoubet, C. P., Schmidt, R., and Goldstein, M. L. (1997). Cluster-science and mission overview. Space Sci. Rev., 79(1-2), 11-32. https://doi.org/10.1023/A:1004923124586
Fu, H. S., Khotyaintsev, Y. V., André, M., and Vaivads, A. (2011). Fermi and betatron acceleration of suprathermal electrons behind dipolarization fronts. Geophys. Res. Lett., 38(16), L16104. https://doi.org/10.1029/2011GL048528

Fu, H. S., Khotyaintsev, Y. V., Vaivads, A., André, M., and Huang, S. Y. (2012a). Occurrence rate of earthward-propagating dipolarization fronts. Geophys. Res. Lett., 39(10), L10101. https://doi.org/10.1029/2012GL051784

Fu, H. S., Khotyaintsev, Y. V., Vaivads, A., André, M., Sergeev, V. A., Huang, S. Y., Kronberg, E. A., and Daly, P. W. (2012b). Pitch angle distribution of suprathermal electrons behind dipolarization fronts: A statistical overview. J. Geophys. Res.: Space Phys., 117(A12), A12221. https://doi.org/10.1029/2012JA018141

Gustafsson, G., André, M., Carozzi, T., Eriksson, A. I., Fälthammar, C.-G., Grard, R., Holmgren, G., Holtet, J. A., Ivchenko, N., ... Wahlund, J. E. (2001). First results of electric field and density observations by CLUSTER EFW based on initial months of operation. Ann. Geophys., 19(10-12), 1219-1240. https://doi.org/10.5194/angeo-19-1219-2001

Huang, S. Y., Zhou, M., Deng, X. H., Yuan, Z. G., Pang, Y., Wei, Q., Su, W., Li, H. M., and Wang, Q. Q. (2012). Kinetic structure and wave properties associated with sharp dipolarization front observed by Cluster. Ann. Geophys., 30(1), 97-107. https://doi.org/10.5194/angeo-30-97-2012

Huang, S. Y., Fu, H. S., Yuan, Z. G., Zhou, M., Fu, S., Deng, X. H., Sun, W. J., Pang, Y., Wang, D. D., ... Yu, X. D. (2015a). Electromagnetic energy conversion at dipolarization fronts: Multispacecraft results. J. Geophys. Res.: Space Phys., 120(6), 4496-4502. https://doi.org/10.1002/2015JA021083

Huang, S. Y., Fu, H. S., Vaivads, A., Yuan, Z. G., Pang, Y., Zhou, M., Khotyaintsev, Y. V., Deng, X. H., André, M., ... Wang, D. D. (2015b). Dawn-dusk scale of dipolarization front in the Earth's magnetotail: multi-cases study. Astrophys. Space Sci., 357(1), 22. https://doi.org/10.1007/s10509-015-2298-3

Liu, J., Angelopoulos, V., Zhou, X. Z., Runov, A., and Yao, Z. H. (2013). On the role of pressure and flow perturbations around dipolarizing flux bundles. J. Geophys. Res.: Space Phys., 118(11), 7104-7118. https://doi.org/10.1002/2013JA019256

Liu, J., Angelopoulos, V., Zhou, X. Z., and Runov, A. (2014). Magnetic flux transport by dipolarizing flux bundles. J. Geophys. Res.: Space Phys., 119(2), 909-926. https://doi.org/10.1002/2013JA019395

Liu, J., Angelopoulos, V., Zhou, X. Z., Yao, Z. H., and Runov, A. (2015). Cross-tail expansion of dipolarizing flux bundles. J. Geophys. Res.: Space Phys., 120(4), 2516-2530. https://doi.org/10.1002/2015JA020997

Li, H. M., Zhou, M., Deng, X. H., Yuan, Z. G., Guo, L. X., Yu, X. D., Pang, Y., and Huang, S. Y. (2015). A statistical study on the whistler waves behind dipolarization fronts. J. Geophys. Res.: Space Phys., 120(2), 1086-1095. https://doi.org/10.1002/2014JA020474

Nakamura, R., Baumjohann, W., Klecker, B., Bogdanova, Y., Balogh, A., Rème, H., Bosqued, J. M., Dandouras, I., Sauvaud, J. A., ... Runov, A. (2002). Motion of the dipolarization front during a flow burst event observed by Cluster. Geophys. Res. Lett., 29(20), 3-1-3-4. https://doi.org/10.1029/2002GL015763

Nakamura, R., Baumjohann, W., Mouikis, C., Kistler, L. M., Runov, A., Volwerk, M., Asano, Y., Vörös, Z., Zhang, T. L., ... Balogh, A. (2004). Spatial scale of highspeed flows in the plasma sheet observed by Cluster. Geophys. Res. Lett., 31(9), L09804. https://doi.org/10.1029/2004GL019558

Ohtani, S., Shay, M. A., and Mukai, T. (2004). Temporal structure of the fast convective flow in the plasma sheet: Comparison between observations and two-fluid simulations. J. Geophys. Res.: Space Phys., 109(A3), A03210. https://doi.org/10.1029/2003JA010002

Pan, D. X., Khotyaintsev, Y. V., Graham, D. B., Vaivads, A., Zhou, X. Z., André, M., Zhou, X. Z., André, M., Lindqvist, P. A., ... Burch, J. L. (2018). Rippled electronscale structure of a dipolarization front. Geophys. Res. Lett., 45(22), 12116-12124. https://doi.org/10.1029/2018GL080826

Pedersen, A., Lybekk, B., André, M., Eriksson, A., Masson, A., Mozer, F. S., Lindqvist, P.-A., DéCréAu, P. M. E., Dandouras, I., ... Whipple, E. (2008). Electron density estimations derived from spacecraft potential measurements on Cluster in tenuous plasma regions. J. Geophys. Res.: Space Phys., 113(A7), A07S33. https://doi.org/10.1029/2007JA012636

Pritchett, P. L., Coroniti, F. V., and Nishimura, Y. (2014). The kinetic ballooning/interchange instability as a source of dipolarization fronts and 
auroral streamers. J. Geophys. Res.: Space Phys., 119(6), 4723-4739. https://doi.org/10.1002/2014JA019890

Pritchett, P. L. (2016). Three-dimensional structure and kinetic features of reconnection exhaust jets. J. Geophys. Res.: Space Phys., 121(1), 214-226. https://doi.org/10.1002/2015JA022053

Rème, H., Aoustin, C., Bosqued, J. M., Dandouras, I., Lavraud, B., Sauvaud, J. A., Barthe, A., Bouyssou, J., Camus, T., ... Sonnerup, B. (2001). First multispacecraft ion measurements in and near the Earth's magnetosphere with the identical Cluster ion spectrometry (CIS) experiment. Ann. Geophys., 19(10-12), 1303-1354. https://doi.org/10.5194/angeo-19-1303-2001

Runov, A., Angelopoulos, V., Sitnov, M. I., Sergeev, V. A., Bonnell, J., McFadden, J. P., Larson, D., Glassmeier, K. H., and Auster, U. (2009). THEMIS observations of an earthward-propagating dipolarization front. Geophys. Res. Lett., 36(14), L14106. https://doi.org/10.1029/2009GL038980

Runov, A., Angelopoulos, V., Zhou, X. Z., Zhang, X. J., Li, S., Plaschke, F., and Bonnell, J. (2011). A THEMIS multicase study of dipolarization fronts in the magnetotail plasma sheet. J. Geophys. Res.: Space Phys., 116(A5), A05216. https://doi.org/10.1029/2010JA016316

Schmid, D., Volwerk, M., Nakamura, R., Baumjohann, W., and Heyn, M. (2011). A statistical and event study of magnetotail dipolarization fronts. Ann. Geophys., 29(9), 1537-1547. https://doi.org/10.5194/angeo-29-1537-2011

Sergeev, V. A., Angelopoulos, V., Gosling, J. T., Cattell, C. A., and Russell, C. T. (1996). Detection of localized, plasma-depleted flux tubes or bubbles in the midtail plasma sheet. J. Geophys. Res. Space Phys., 101(A5), 10817-10826. https://doi.org/10.1029/96JA00460

Sonnerup, B. U. Ö., and Scheible, M. (1998). Minimum and maximum variance analysis. In G. Paschmann, et al. (Eds.), Analysis Methods for Multi-Spacecraft
Data (pp. 185-22). Bern, Switzerland: International Space Science Institute.

Vapirev, A. E., Lapenta, G., Divin, A., Markidis, S., Henri, P., Goldman, M., and Newman, D. (2013). Formation of a transient front structure near reconnection point in 3-D PIC simulations. J. Geophys. Res.: Space Phys., 118(4), 1435-1449. https://doi.org/10.1002/jgra.50136

Wang, Y., Zhou, M., and Deng, X. H. (2015). Statistical study on the suprathermal electrons properties around dipolarization fronts in Earth's magnetotail. Sci. China Technol. Sci., 58(6), 961-966. https://doi.org/10.1007/s11431-0155830-3

Zhou, M., Ashour-Abdalla, M., Deng, X. H., Schriver, D., El-Alaoui, M., and Pang, $Y$. (2009). THEMIS observation of multiple dipolarization fronts and associated wave characteristics in the near-Earth magnetotail. Geophys. Res. Lett., 36(20), L20107. https://doi.org/10.1029/2009GL040663

Zhou, M., Deng, X. H., Ashour-Abdalla, M., Walker, R., Pang, Y., Tang, C. L., Huang, S. Y., El-Alaoui, M., Yuan, Z. G., and Li, H. M. (2013). Cluster observations of kinetic structures and electron acceleration within a dynamic plasma bubble. J. Geophys. Res.: Space Phys., 118(2), 674-684. https://doi.org/10.1029/2012JA018323

Zhou, M., Ni, B. B., Huang, S. Y., Deng, X. H., Ashour-Abdalla, M., Nishimura, Y., Yuan, Z. G., Pang, Y., and Li, H. M. (2014). Observation of large-amplitude magnetosonic waves at dipolarization fronts. J. Geophys. Res.: Space Phys., 119(6), 4335-4347. https://doi.org/10.1002/2014JA019796

Zhou, M., Ashour-Abdalla, M., Deng, X. H., Pang, Y., Fu, H. S., Walker, R., Lapenta, G., Huang, S. Y., Xu, X. J., and Tang, R. X. (2017). Observation of threedimensional magnetic reconnection in the terrestrial magnetotail. $J$. Geophys. Res.: Space Phys., 122(9), 9513-9520. https://doi.org/10.1002/2017JA024597 